\title{
Optimal Placement of CRNs in Manned/ Unmanned Aerial Vehicle Cooperative Engagement System
}

\author{
Yun Zhong ${ }^{1}$, Peiyang Yao ${ }^{1}$, Lujun Wan $^{2}$ and Yeming Xiong ${ }^{3}$ \\ ${ }^{1}$ Information and Navigation College, Air Force Engineering University \\ Xi'an, Shaanxi 710077 - China \\ [e-mail: 718227697@qq.com] \\ ${ }^{2}$ Air Traffic Control and Navigation College, Air Force Engineering University \\ Xi'an, Shaanxi 710051 - China \\ [e-mail: pandawlj@126.com] \\ ${ }^{3}$ Financial Planning Department, Xi'dian University \\ Xi'an, Shaanxi 710071 - China \\ [e-mail: 18629615032@163.com] \\ *Corresponding author: Yun Zhong
}

Received September 2, 2017; revised April 24, 2018; revised August 28, 2018; accepted September 24, 2018; published January 31, 2019

\begin{abstract}
Aiming at the optimal placement of communication relay nodes (OPCRN) problem in manned/unmanned aerial vehicle cooperative engagement system, this paper designed a kind of fully connected broadband backbone communication topology. Firstly, problem description of OPCRN was given. Secondly, based on problem analysis, the element attributes and decision variables were defined, and a bi-level programming model including physical layer and logical layer was established. Thirdly, a hierarchical artificial bee colony (HABC) algorithm was adopted to solve the model. Finally, multiple sets of simulation experiments were carried out to prove the effectiveness and superiority of the algorithm.
\end{abstract}

Keywords: Communication relay nodes (CRNs), optimal placement, fully connected broadband backbone structure, bi-level programming model, hierarchical artificial bee colony (HABC) 


\section{Introduction}

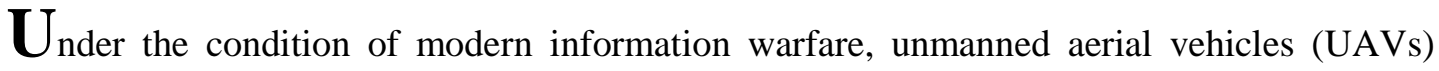
possessing the capacity of situation awareness, independent decision-making and cooperative attack, have been widely used. UAVs can take the advantage of agile and flexible tactics, strong continuous combat capacity and low life cycle cost, to complete combat mission under the extreme miserable combat environment [1]. However, because the intelligent level of UAVs is not high, it is hard for UAVs to cope with the battlefield environment with high uncertainty and strong emergent. Hence, via combining UAVs with manned aerial vehicles (MAVs), and giving full play to the command art and combat experience of commanders, $\mathrm{MAV/UAV}$ cooperative engagement system will have complementary advantages and achieve the maximum combat effectiveness [2].

In MAV/UAV cooperative engagement system, combat planning department usually divides tasks with similar geographical location and resource demand into the same task cluster, and tasks in each task cluster will be performed by a number of MAVs and UAVs which form a certain task cluster coalition [3]. In the actual battlefield environment, due to communication capability limitations of MAVs and UAVs, it is impossible for different MAV/UAV task cluster coalitions to communicate with each other directly. Therefore, unmanned airships are usually used as communication relay nodes (CRNs), and through rational placement of these high-altitude CRNs, a fully connected communication network among different MAV/UAV task cluster coalitions can be realized [4].

This paper focuses on the problem of designing the placement scheme of CRNs to satisfy the demand of MAV/UAV cooperative engagement system. Section 2 reviews the previous work in communication and network problem in MAV/UAV cooperative engagement system. Section 3 gives a detailed description about the problem of providing communication relay services to MAV/UAV cooperative engagement system by CRNs. In Section 4, we establish a typical bi-level model including physical layer and logical layer and adopt a novel hierarchical artificial bee colony (HABC) algorithm to solve the model in Section 5. Section 6 provides a numerical example to prove the effectiveness and superiority of the algorithm, and finally, Section 7 gives a summary and suggestion for future research.

\section{Related Work}

To the best of our knowledge, there have been few studies on the OPCRN problem of MAV/UAV cooperative engagement, Ref. [5] proposed a design framework for MAV/UAV communication system, and it did not go deep into the model level. The main research area addressing the OPCRN problem mainly included robot system, multi-agent system, wireless sensor networks (WSNs) system and multi-UAV system, and these research results could provide reference for this paper.

In robot system, Ref. [6] presented a maintaining strategy for end-to-end communication links between multi-robots performing surveillance, reconnaissance and target search tasks, and then the authors proposed the construction of a Radio Signal Strength Map (RSSP), and designed reactive controllers to gurantee the communication link maintenance. In multi-agent system, Ref. [7] proposed a complex network model for the system, and designed evolutionary optimized networks, which took consensus and synchronization as the objective function. Simulation results showed that the convergence speed was faster than that of random regular 
networks, and the results revealed that the connectivity had a great impact on network performance.

In WSNs system, aiming at the important limitation of two genetic algorithms (NSGA-II, SPEA2) in optimizing traditional static WSNs by adding relay nodes, i.e., the computation time was high, Ref. [8] parallelized both algorithms using OpenMP to reduce the computation time, and achieved a quite good efficiency. Ref. [9] aimed at deploying a minimum number of relay nodes to realize the maximum coverage, it presented different polynomial-time approximation algorithms to solve different problems with different coverage requirements, and it proved the mathematical complexity of the algorithms.

In multi-UAV system, Ref. [10] pointed out that the communication system of multi-UAV was an important factor influencing the system operational effectiveness, and gave a detailed account of the development status of several typical communication systems. From the perspective of the relationship between communication topology and distributed auction algorithm performance, Ref. [11] proved convergence and optimality of the algorithm under the condition of a changing topology, i.e., communication topology structure had a great effect on algorithm convergence speed. Ref. [12] took communication coverage and communication service quality as optimization objectives, and adopted distributed dynamic optimization method based on game learning to realize the optimal placement of network node. Aiming at the problem of establishing full connectivity and meeting communication traffic constraints between disconnected ground clusters by deploying as few as CRNs at appropriate places, Ref. [13] transformed the problem into a constrained clustering problem and adopted the deterministic annealing clustering algorithm to solve it. To optimize the connectivity of a wireless network, Ref. [14] summarized four types of network connectivity, and addressed the problem of utilizing CRNs to improve the communication condition, after that, targeted algorithms were proposed to determine the deployment and movement of CRNs.

Due to the complexity of battlefield environment, it is difficult for all platforms to master global information, while a consistent understanding of battlefield situation is the precondition to realize self-synchronization engagement [15]. In fact, self-synchronization in task level is closely associated with topology in communication level [16], i.e., a usable, credible and controllable communication topology is the basis of converting information consistency to decision consistency. Hence, the communication relationship of MAV/UAV cooperative engagement system has a direct impact on combat effectiveness, and it is vital to design a reasonable communication relationship for the system.

Actually, in the field of communication relay planning, the optimization factors generally included number of relay nodes [17], connectivity [18,19], capacity [20,21], and safety [22]. However, few of literatures once in consideration of comprehensive objectives, besides, there were a few differences between MAV/UAV cooperative engagement system and other fields, i.e., (1) the communication capacities of MAVs and UAVs are different, (2) the dynamic character of UAVs is stronger than that of MAVs, (3) the communication functions of MAVs and UAVs are different. Hence, it is of great significance to carry out research on OPCRN problem in MAV/UAV cooperative engagement system according to system characteristics.

\section{Problem description}

According to the different task granularities, a typical MAV/UAV cooperative engagement system can be divided into two-layer coalitions, i.e., MAV/UAV task cluster coalition and MAV/UAV task coalition, thus simplify the scale of problem.

To illustrate the problem, we take Fig. 1 as an example. For a typical task set, by adopting 
a clustering algorithm, all tasks can be clustered into different task clusters according to their geographical distance and resource demand. In order to perform a particular task cluster, some MAVs and UAVs in the platform set will form a corresponding task cluster coalition by adopting an intelligent optimization algorithm, and the formation principle is to maximize decision-making capability cost and resource capability cost. While for tasks in each task cluster, by adopting an intelligent optimization algorithm, some UAVs in the particular task cluster coalition will form corresponding task coalitions to perform them, and the formation principle is to minimize the task completion time and maximize the task completion quality.

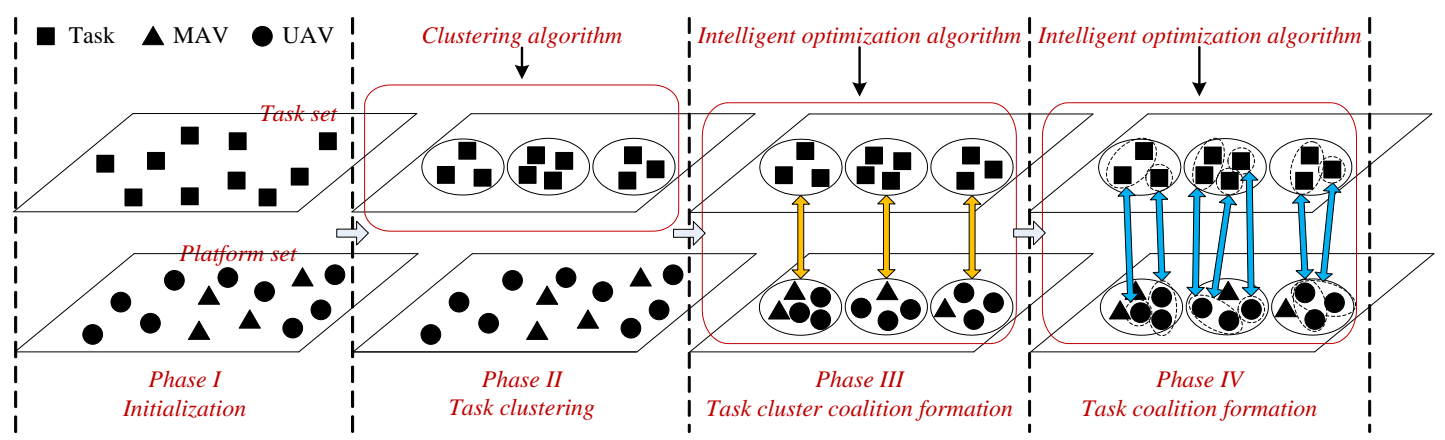

Fig. 1. Phased-assignment of tasks in MAV/UAV cooperative engagement system

From the above analysis, we can see that MAV/UAV cooperative engagement system is a three-layer system, respectively, $C_{0}$ (MAV/UAV cooperative engagement system itself), $C_{1}$ (MAV/UAV task cluster coalition) and $C_{2}$ (MAV/UAV task coalition). Fig. 2 shows the relationship of three-layer structure in MAV/UAV cooperative engagement system.

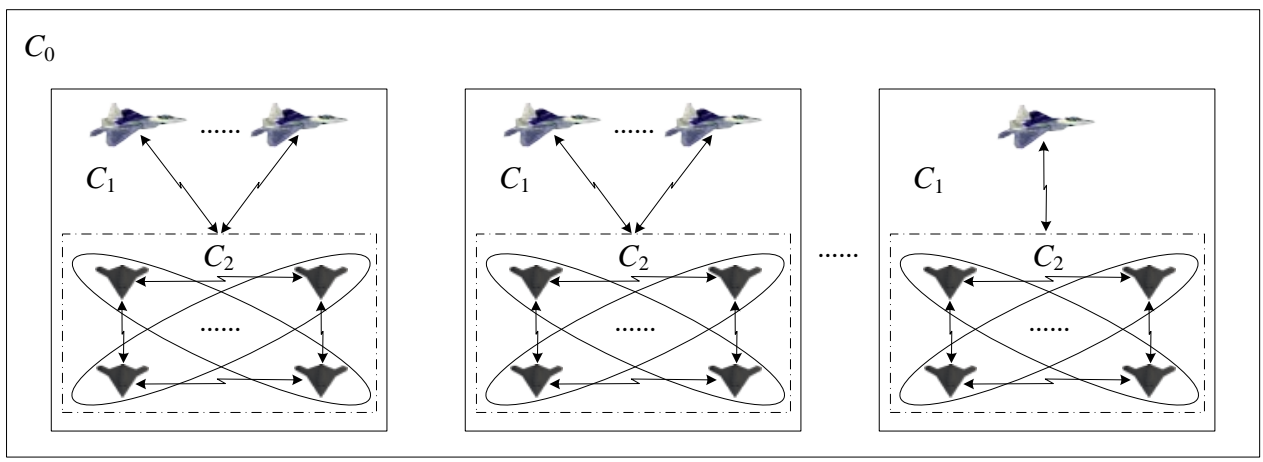

$C_{0}:$ MAV/UAV cooperative engagement system $\quad C_{1}:$ MAV/UAV task cluster coalition

$C_{2}:$ MAV/UAV task coalition

Fig. 2. The relationship of three-layer structure in MAV/UAV cooperative engagement system

Considering that the dynamism, isomerization and wide distribution characteristic of UAVs in cooperative engagement system, meanwhile, the communication capability of MAVs is stronger, and MAVs can always keep them over a relatively fixed location, thus, it is easy for MAVs to establish stable or approximately stable links. Consequently, when building communication topology of cooperative engagement system, MAVs are taken as gateway nodes, and through communication relay, a fully connected broadband backbone network (BN) among MAVs is formed. UAVs in each task cluster coalition communicate to MAVs and other UAVs in respective task cluster coalition by adopting opportunistic access method, and 
in this way can realize cooperative share of status information, intelligence information, command and control (C2) information and attack information [23].

As shown in Fig. 3 and Fig. 4, they reveal that how CRNs provide communication services and what logical network of cooperative engagement system is like. In this paper, in order to reduce the complexity of the problem, we assume that the flight altitude of all CRNs remains unchanged.

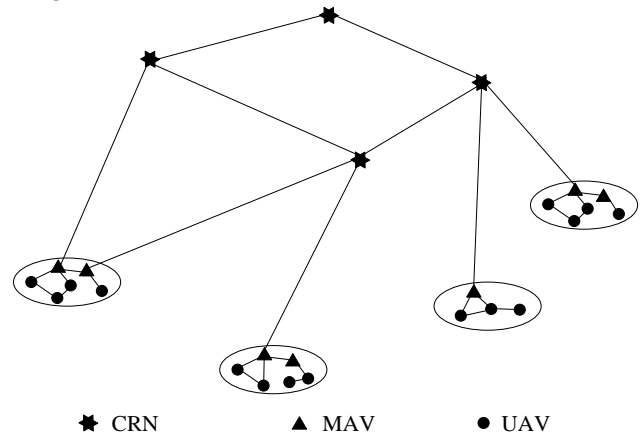

Fig. 3. CRNs provide communication services

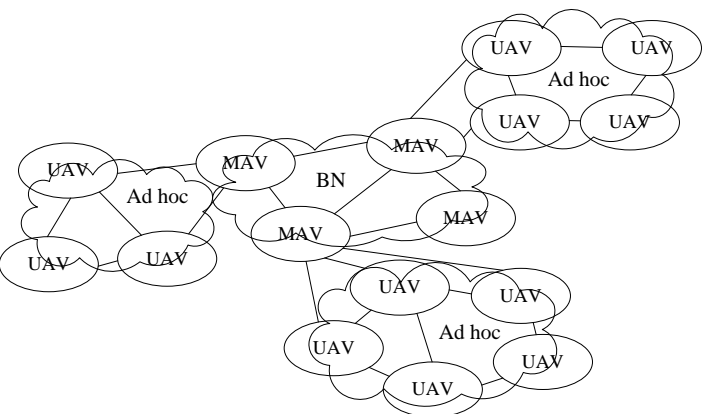

Fig. 4. Logical network of engagement system

\section{Problem modeling}

\subsection{Definition of element attributes and decision variables}

First of all, it defines the element attributes and decision variables of OPCRN problem, within which element attributes contain task cluster coalition set, MAV set and CRN set, while decision variables contain MAV-task cluster relationship decision variable, MAV-CRN relationship decision variable and CRN-CRN relationship decision variable. Next, a detailed introduction is given.

(1) Task cluster coalition set TCC $=\left\{t c c_{1}, t c c_{2}, \cdots, t c c_{N_{t c c}}\right\}$, where $N_{t c c}$ is the number of task cluster coalitions.

(2) MAV set $M=\left\{m_{1}, m_{2}, \cdots, m_{N_{m}}\right\}$, where $N_{m}$ is the number of MAVs, and coordinate position of the $j$ th MAV i.e., $m_{j}$, in $X-Y$ coordinate system is $a_{j}=\left(x_{m_{j}}, y_{m_{j}}\right)$.

(3) CRN set $Z J=\left\{z j_{1}, z j_{2}, \cdots, z j_{N_{z j}}\right\}$, where $N_{z j}$ is the number of CRNs, and projection coordinate position of the $k$ th CRN, i.e., $z j_{k}$, in $X-Y$ coordinate system is $b_{k}=\left(x_{z j_{k}}, y_{z j_{k}}\right)$.

(4) $\delta_{i j}$ is the decision variable indicating whether $m_{j}$ belongs to $t c c_{i}, \delta_{i j}=1$ represents $m_{j}$ belongs to $t c c_{i}$, while $\delta_{i j}=0$ represents not.

(5) $\xi_{j k}$ is the decision variable indicating whether $m_{j}$ is covered by $z j_{k}, \xi_{j k}=1$ represents $m_{j}$ is covered by $z j_{k}$, while $\xi_{j k}=0$ represents not.

(6) $\zeta_{i k}$ is the decision variable indicating whether $t c c_{i}$ transmits information to other task cluster coalitions through $z j_{k}, \zeta_{i k}=1$ represents yes, while $\zeta_{i k}=0$ represents not. $\zeta_{i k}$ is a logical layer decision variable, the necessary condition of $\zeta_{i k}=1$ is $\sum_{j=1}^{N_{m}} \delta_{i j} \xi_{j k}=1$. 
(7) $\varsigma_{k \rightarrow k^{\prime}}^{i \rightarrow i^{\prime}}$ is the decision variable indicating that when $t c c_{i}$ transmits information to $t c c_{i^{\prime}}$, whether there is a transmission path from $z j_{k}$ to $z j_{k^{\prime}}, \varsigma_{k \rightarrow k^{\prime}}^{i \rightarrow i^{\prime}}=1$ represents yes, while $\varsigma_{k \rightarrow k^{\prime}}^{i \rightarrow i^{\prime}}=0$ represents not. $\varsigma_{k \rightarrow k^{\prime}}^{i \rightarrow i^{\prime}}$ is also a logical layer decision variable, the necessary condition of $\varsigma_{k \rightarrow k^{\prime}}^{i \rightarrow i^{\prime}}=1$ is that, 1) $\left.\zeta_{i k}=1,2\right) \zeta_{i^{\prime} k^{\prime}}=1$, and 3) there exists a physical link between $z j_{k}$ and $z j_{k^{\prime}}$, either one-hop or multi-hops.

\subsection{Physical layer}

\subsubsection{Constraint analysis}

The placement of CRNs has to satisfy the following two conditions, the first one is that, for any task cluster coalition $t c c_{i}$, there must have at least one MAV $m_{j}$ locating within the relay range of $z j_{k}$, the second one is that, for any CRN $z j_{k^{\prime}}$, there must have at least one another CRN $z j_{k}$ can be connected to. Fig. 5 shows the placement situation of CRNs satisfying the above two conditions simultaneously.

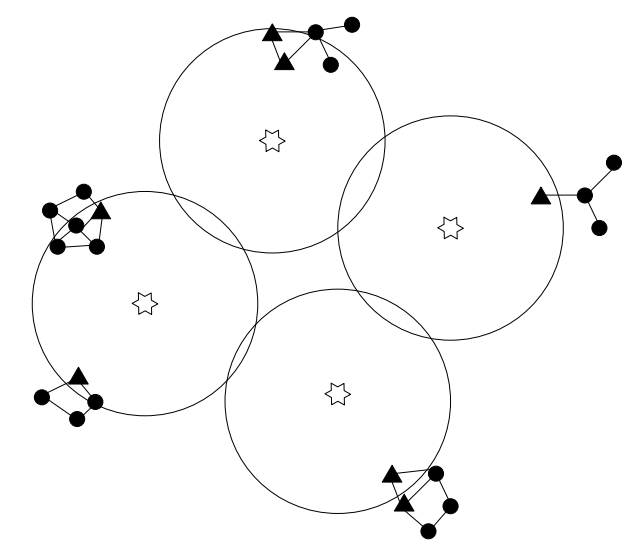

Fig. 5. The placement situation of CRNs

So, communication connection constraint of OPCRN problem is

$$
\begin{gathered}
\min _{\delta_{i j} \neq 0} \delta_{i j}\left\|a_{j}-b_{k}\right\| \leq R_{1}, \quad \forall j=1,2, \cdots, N_{m} ; \exists k=1,2, \cdots, N_{z j} \\
\max \min \left\|b_{k^{\prime}}-b_{k}\right\| \leq R_{2}, \quad \exists k, k^{\prime}=1,2, \cdots, N_{z j}, k \neq k^{\prime}
\end{gathered}
$$

where $\|\cdot\|$ is Euclidean distance computing operator, $R_{1}$ is communication range between MAVs and CRNs, $R_{2}$ is communication range between CRNs and CRNs. The first constraint guarantees that any MAV/UAV task cluster coalition can be covered by at least one CRN, the second constraint guarantees that any CRN can be connected to at least one another CRN.

Once Formula (1) and Formula (2) are established, a fully connected broadband backbone structure among different task cluster coalitions will be formed.

4.2.2 Objective function analysis

Considering the high value of CRNs, it is necessary to use as few CRNs as possible to provide communication relay services for MAV/UAV cooperative engagement system, and objective function of the model is defined as

$$
\min N_{z j}
$$


To sum up, this paper establishes the mathematical model of physical layer as

$$
\begin{aligned}
& \min N_{z j} \\
& \text { s.t. }\left\{\begin{array}{l}
\min _{\delta_{i j} \neq 0} \delta_{i j}\left\|a_{j}-b_{k}\right\| \leq R_{1}, \quad \forall j=1,2, \cdots, N_{m} ; \exists k=1,2, \cdots, N_{z j} \\
\max \min \left\|b_{k^{\prime}}-b_{k}\right\| \leq R_{2}, \quad \exists k, k^{\prime}=1,2, \cdots, N_{z j}, k \neq k^{\prime}
\end{array}\right.
\end{aligned}
$$

\subsection{Logical layer}

\subsubsection{Constraint analysis}

Constraints in logical layer are closely related to relationship between logical layer decision variables and phisical layer decision variables. The details are as follows.

(1) Considering the limit of communication link bandwidth, communication bandwidth provided by each CRN must not be greater than its communication bandwidth threshold, i.e.,

$$
\sum_{i=1}^{N_{t c c}} \zeta_{i k} B_{t c c_{i}} \leq B_{\max }, \quad \forall k=1,2, \cdots, N_{z j}
$$

where $B_{t c c_{i}}$ is communication bandwidth demand of $t c c_{i}, B_{\max }$ is communication bandwidth threshold of all CRNs.

(2) Considering the relationship among $\delta_{i j} 、 \xi_{j k}$ and $\zeta_{i k}$, there is

$$
\sum_{j=1}^{N_{m}} \delta_{i j} \xi_{j k} \geq \zeta_{i k}, \quad \forall i=1,2, \cdots, N_{t c c} ; \forall k=1,2, \cdots, N_{z j}
$$

established.

(3) In addition, the existence of a logical link is based on the premise that there exists a physical link, hence,

$$
\operatorname{Bool}\left(\left\|b_{k^{\prime}}-b_{k}\right\| \leq R_{2}\right) \geq \varsigma_{k \rightarrow k^{\prime}}^{i \rightarrow i^{\prime}}, \quad \forall i, i^{\prime}=1,2, \cdots, N_{t c c}, i \neq i ; \forall k, k^{\prime}=1,2, \cdots, N_{z j}, k \neq k^{\prime}
$$

where $\operatorname{Bool}(\cdot)$ is Boolean function.

\subsubsection{Objective function analysis}

Objective functions of logical layer are defined as the reciprocal of average communication service quality and the average number of hops.

(1) In general, communication service qualities provided by CRNs to each MAV/UAV task cluster coalition are different, the values of which depend on received transmitting power of communication antenna by MAVs, and MAVs' allocation situation, i.e., the value of $\zeta_{i k}$ and $S_{k \rightarrow k^{\prime}}^{i \rightarrow i^{\prime}}$. The detailed solving process is as follows.

Assuming that the wireless communication channel is Rician channel [24,25], hence, signal power received by $m_{j}$ from $z j_{k}$ is

$$
P_{j}^{k}=\frac{\xi_{j k} \theta P^{k}}{\left\|h^{2}+R_{1}^{2}\right\|^{\alpha}}
$$

where $h$ is the height of $z j_{k}, P^{k}$ is the transmitting power of $z j_{k}, \theta$ is a constant, and $\alpha$ is path attenuation factor.

If $m_{j}$ is covered by multiple CRNs simultaneously, to simplify, when communication interference is not considered, the effective signal power received by $m_{j}$ is

$$
P_{j}=\sum_{k=1}^{N_{z j}} \zeta_{i k} P_{j}^{k}
$$

Then, communication service quality obtained by $t c c_{i}$ is the maximum signal power received by all MAVs in $t c c_{i}$, i.e., 


$$
P(i)=\max \left(\delta_{i 1} P_{1}, \delta_{i 2} P_{2}, \cdots, \delta_{i N_{m}} P_{N_{m}}\right)
$$

Hence, one of objective functions for logical layer is the average communication service quality obtained by all MAV/UAV task cluster coalitions, i.e.,

$$
\max \bar{P}=\max \frac{1}{N_{t c c}} \sum_{i=1}^{N_{t c c}} P(i)
$$

(2) To maximize the information transmission efficiency in MAV/UAV cooperative engagement system, it is necessary to minimize the average number of hops from one MAV/UAV task cluster coalition to others. Firstly, calculate the number of hops from $t c c_{i}$ to all of other $t c c_{i^{\prime}}$, calculating formula is

$$
\sum_{k=1}^{N_{z j}} \zeta_{i k}+\sum_{k^{\prime}=1}^{N_{z j}} \varsigma_{k \rightarrow k^{\prime}}^{i \rightarrow i^{\prime}}+\sum_{k=1}^{N_{z j}} \zeta_{i^{\prime} k}, \quad \forall i, i^{\prime}=1,2, \cdots, N_{t c c}, i \neq i^{\prime}
$$

Then, another objective functions for logical layer is the average number of hops from one MAV/UAV task cluster coalition to others, i.e.,

$$
\min \bar{Q}=\min \frac{N_{t c c}\left(N_{t c c}-1\right)}{2} \sum_{i=1}^{N_{t c c}}\left(\sum_{k=1}^{N_{z j}} \zeta_{i k}+\sum_{k^{\prime}=1}^{N_{z j}} \varsigma_{k \rightarrow k^{\prime}}^{i \rightarrow i^{\prime}}+\sum_{k=1}^{N_{z j}} \zeta_{i^{\prime} k}\right)
$$

In conclusion, the mathematical model of logical layer is established as

$$
\begin{array}{ll}
\min \left(\frac{1}{\bar{P}}, \bar{Q}\right) & \forall k=1,2, \cdots, N_{z j} \\
\text { s.t. } \begin{cases}\sum_{i=1}^{N_{t c c}} \zeta_{i k} B_{t c c_{i}} \leq B_{\max }, & \forall i=1,2, \cdots, N_{t c c} ; \forall k=1,2, \cdots, N_{z j} \\
\sum_{j=1}^{N_{m}} \delta_{i j} \xi_{j k} \geq \zeta_{i k}, & \forall i, i^{\prime}=1,2, \cdots, N_{t c c}, i \neq i ; \forall k, k^{\prime}=1,2, \cdots, N_{z j}, k \neq k^{\prime} \\
\operatorname{Bool}\left(\left\|b_{k^{\prime}}-b_{k}\right\| \leq R_{2}\right) \geq \zeta_{k \rightarrow k^{\prime}}^{i \rightarrow i^{\prime}},\end{cases}
\end{array}
$$

In short, the upper planning objective of OPCRN problem is to minimize the number of CRNs, and the lower planning objective of OPCRN problem is to minimize the reciprocal of the average communication service quality and the average number of hops.

\section{Using HABC algorithm to solve OPCRN problem}

\subsection{Standard ABC algorithm}

$\mathrm{ABC}$ algorithm is a kind of intelligent optimization algorithm whose working principle is simulating the harvest behavior of bees [26], and it divides bees into employed bees, onlooker bees and scout bees, which can be transformed under certain condition. In ABC algorithm, a honey source position represents a solution, and the amount of honey source represents the quality of solutions. Generally speaking, the computational process of ABC algorithm is as follows.

(1) Initialization phase. If the number of population is $N_{Z Q}$, the dimension of honey source is $N_{W}$, then every honey source position can be generated randomly in feasible interval. The calculating formula is

$$
x_{i j}=x_{j}^{\min }+\operatorname{rand}(0,1) \times\left(x_{j}^{\max }-x_{j}^{\min }\right)
$$

where $x_{j}^{\max }$ and $x_{j}^{\min }$ is the upper and lower bound of honey source position value, rand $(0,1)$ represents a random number evaluating at 0 to 1 . 
(2) Employed bee phase. Employed bees carry out local search near honey source, i.e., generate new honey source position according to Formula (16),

$$
z_{i j}{ }^{\prime}=z_{i j}+\operatorname{rand}(-1,1) \times\left(z_{i j}-z_{k j}\right)
$$

where $i \neq k$, and $z_{k j}$ is selected randomly from all honey sources, rand $(-1,1)$ represents a random number evaluating at -1 to 1 . Compare new honey source with old honey source, if the former one is superior to the latter one, then replace old honey source with new honey source, otherwise, remain unchanged.

(3) Onlooker bee phase. After all employed bees have completed local search, position information of honey sources is shared to onlooker bees by employed bees' swing dance. Then, onlooker bees adopt tournament selection operator to select a honey source, i.e., randomly select two honey sources $\boldsymbol{Z}_{1}$ and $\boldsymbol{Z}_{2}$, then compare the corresponding fitness values, $F_{1}$ and $F_{2}$, if there is

$$
F_{1} \prec F_{2}
$$

established, then select $\boldsymbol{Z}_{1}$ to update according to Formula (16), else, select $\boldsymbol{Z}_{2}$ to carry out the same operation, where $\prec$ represents Pareto non-inferiority.

(4) Scout bee phase. After Limit iterations, if the fitness value of a certain honey source position has not improved, then the corresponding employed bee will be transformed into scout bee, old honey source will be abandoned and new honey source will be generated according to Formula (15).

When adopting $\mathrm{ABC}$ algorithm to solve multi-objective problem, sorting result of fitness value is achieved by non-dominated ranking and crowding distance calculation, then Pareto front will be obtained.

\subsection{HABC algorithm}

\subsubsection{Basic procedure}

Considering that OPCRN problem is a multiple decision variables bi-level programming problem, it is an important concern for encoding and decoding. For convenience, this paper does not consider decision variables defined in Section 2.1 as code elements, while consider coordinate positions of CRNs as code elements. Take population $\mathbf{Z}$ for example, its row number is $N_{z Q}$, each row represents a solution, its column number is $N_{W}$, in which, odd columns represent horizontal coordinates and even columns represent vertical coordinates.

To simplify the problem, $\delta_{i j}$ is predetermined, and in specific population $\boldsymbol{Z}$, by calculating the relative positional relationship between MAVs and CRNs, or between CRNs and CRNs, the value of $\xi_{j k}$ and $\zeta_{k \rightarrow k^{\prime}}^{i \rightarrow i^{\prime}}$ is determined, while the value of $\zeta_{i k}$ depends on $\xi_{j k}$ and setting in specific solving process.

Based on the game theory of dual decision makers, a novel HABC algorithm is proposed to solve OPCRN problem. During the evolution of the algorithm, upper and lower layers are optimized according to each other's evolutionary result, with the increasing of iterations, the whole system is gradually in equilibrium.

The basic procedure of HABC algorithm is as follows.

Step 1 Initialize upper planning population $\boldsymbol{Z}$, set iteration number (assuming that outer iteration number is equivalent to iteration number of $\mathrm{ABC}$ algorithm). The upper and lower bounds of odd column values are the upper and lower bounds of all MAVs' horizontal coordinates, while the upper and lower bounds of even column values are the upper and lower 
bounds of all MAVs' vertical coordinates.

Step 2 By taking $\mathbf{Z}$ as input, optimal solution of lower level planning $W^{*}$ is obtained through adopting $\mathrm{ABC}$ algorithm.

Step 3 By taking $W^{*}$ as input, optimal solution of lower level planning $Z^{*}$ is obtained through adopting $\mathrm{ABC}$ algorithm.

Step 4 If it is the first iteration, constraint handling for $Z^{*}$ and $W^{*}$ is carried out, then $Z_{1}^{*}$ and $\boldsymbol{W}_{1}^{*}$ are obtained. The fitness values of $\boldsymbol{Z}_{1}^{*}$ and $\boldsymbol{W}_{1}^{*}$ are calculated respectively, then non-dominated ranking and crowding distance calculation for all $2 N_{Z Q}$ fitness values are carried out, and the corresponding solutions of the former $N_{Z Q}$ fitness values are selected to form new population $\mathbf{Z}$. If it is not the first iteration, constraint handlings for $\mathbf{Z}, \mathbf{Z}^{*}$ and $\mathbf{W}^{*}$ are carried out, then $\boldsymbol{Z}_{1}, \mathbf{Z}_{1}^{*}$ and $\boldsymbol{W}_{1}^{*}$ are obtained. The fitness values of $\boldsymbol{Z}_{1}, \mathbf{Z}_{1}^{*}$ and $\boldsymbol{W}_{1}^{*}$ are calculated respectively, then non-dominated ranking and crowding distance calculation for all $3 N_{Z Q}$ fitness values are carried out, and the corresponding solutions of the former $N_{Z Q}$ fitness values are selected to form new population $\mathbf{Z}$.

Step 5 If it has reached the maximum iteration number Iter, $\boldsymbol{Z}_{1}$ is obtained after constraint handling for $\boldsymbol{Z}$, and then the fitness value of $\boldsymbol{Z}_{1}$ is calculated. Afterwards, non-dominated ranking and crowding distance calculation for all $N_{Z Q}$ fitness values are carried out, and the population corresponding to optimal fitness values is selected as optimal solutions, otherwise, return to Step 2.

As is shown in Fig. 6, is the cycle game of population in HABC algorithm.

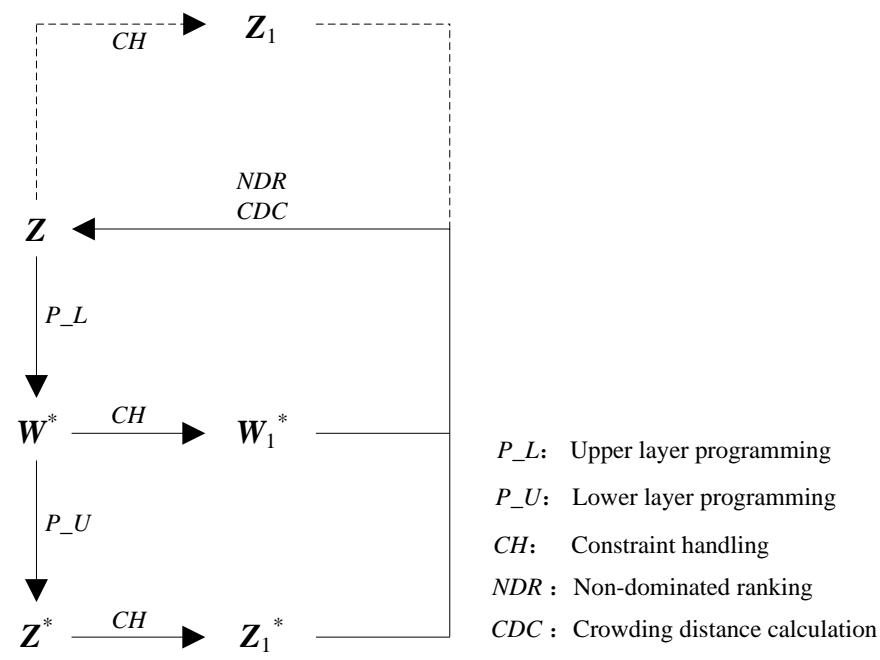

Fig. 6. The cycle game of population in HABC algorithm

\subsubsection{The upper bound value of $N_{W}$ and constraint handling}

This section determines the upper bound value of $N_{W}$ and constraint handling method for HABC algorithm.

(1) The upper bound value of $N_{W}$. As the code elements are coordinates of CRNs, during the running time of HABC algorithm, the number of CRNs has been constantly changing. In addition, a large $N_{W}$ will lead to the increase of algorithm search space, and reduce the 
solving speed of the algorithm, while a small $N_{W}$ will increase the difficulty to satisfy constraint conditions. Therefore, it is necessary to set a reasonable $N_{W}$ value.

This paper sets the upper bound value of $N_{W}$ to be 1.5 times higher than the number of CRNs needed to realize the minimum cover of all MAVs.

(2) Constraint handling. OPCRN problem is a multi-constraints optimization problem, at present, non-feasible solution treatment technology in combinational optimization problem mainly includes two categories [27]. The first one is to reserve non-feasible solutions, and then defines constraint violation function, constraint penalty function, or adopts constraineddominance technology to deal with non-feasible solutions. The second one takes non-feasible solutions as input, and converts them into feasible solutions by adopting a series of operators. The selection of treatment technology depends on the structure of solution space, if feasible solutions take a larger percentage, then the former one is more suitable, while if non-feasible solutions take a larger percentage, then the latter one is more suitable. Considering that it is difficult to estimate the solution space of OPCRN problem, from the perspective of algorithm feasibility, the latter technology is adopted. The detailed steps are as follows.

Step 1 Redundancy removal. Delete CRNs which do not affect communication connection, the criteria is that after deleting a certain CRN, the following two conditions are still valid. 1) The value of $\delta_{i j} \xi_{j k}$ does not change before and after the deletion of a certain CRN. 2) The value of $S_{k \rightarrow k^{\prime}}^{i \rightarrow i^{\prime}}$ does not change before and after the deletion of a certain CRN.

Step 2 The first stage of inadequate supplement operation, judge that whether all task cluster coalitions are covered by at least one CRN, i.e., judge that if $\sum_{j=1}^{N_{m}} \sum_{k=1}^{N_{z j}} \delta_{i j} \xi_{j k} \geq 1$ is established. If there is, go to Step 3, if there is not, select a task cluster coalition $t c c_{i}$, which satisfies $\sum_{j=1}^{N_{m}} \sum_{k=1}^{N_{z j}} \delta_{i j} \xi_{j k}=0$, and select a MAV $m_{j}$ satisfying $\delta_{i j}=1$ randomly, then center at the position of $m_{j}$ to make a circle with radius $R_{1}$, and take any one position in the newly generated circle as the coordinate of added CRN, until there is $\sum_{j=1}^{N_{m}} \sum_{k=1}^{N_{i j}} \delta_{i j} \xi_{j k} \geq 1$ established.

Step 3 The second stage of inadequate supplement operation, judge that whether undirected graph which is formed by all CRNs is fully connected, i.e., judge that whether there is $\sum_{k, k^{\prime}=1}^{N_{z j}} \varsigma_{k \rightarrow k^{\prime}}^{i \rightarrow i^{\prime}} \geq 1$ established. If there is, go to Step 4, if there is not, select any two of connected components, and then choose the nearest two CRNs in respective connected components, make a line segment $s$ between the two selected CRNs. Start from any one of $\mathrm{CRN}$, take the point on the line segment $S$ as the central coordinate of circle for added CRN with step size $\left(0,2 R_{2}\right)$, until there is $\sum_{k, k^{\prime}=1}^{N_{z j}} s_{k \rightarrow k^{\prime}}^{i \rightarrow i^{\prime}} \geq 1$ established.

Step 4 Redo the redundancy removal operation until all constraints are satisfied.

\section{Simulation experiment}

To verify the effectiveness and superiority of HABC algorithm, this paper carries out three groups of simulation experiments by using MATLAB 2010a as simulation platform in Lenovo computer, of which CPU configuration is Intel Dual-Core $3.06 \mathrm{GHz}$, to compare HABC algorithm with hierarchical particle swarm optimization (HPSO) algorithm. 
In the setting of scenario parameters, set $N_{\text {tcc }}=7, N_{m}=13, R_{1}=12$ in Group 1 simulation experiment and Group 3 simulation experiment, and $R_{1}$ is set to be a variable in Group 2 simulation experiment, set $R_{2}=2 R_{1}=24, h=20, P_{0}=10, \theta=1, \alpha=2$. Besides, MAVs' allocation situation is that, $m_{1}$ and $m_{2}$ belong to $t c c_{1}, m_{3}$ and $m_{4}$ belong to $t c c_{2}, m_{5}$ belongs to $t c c_{3}, m_{6}, m_{7}$ and $m_{8}$ belong to $t c c_{4}, m_{9}$ and $m_{10}$ belong to $t c c_{5}, m_{11}$ and $m_{12}$ belong to $t c c_{6}, m_{13}$ belongs to $t C_{7}$.

In the setting of HABC algorithm, set $N_{z q}=20$, Iter $=30$, Limit $=5$. Table 1 shows the coordinate information of MAVs.

Table 1. The coordinate information of MAVs

\begin{tabular}{|c|c|c|c|c|c|}
\hline SN & Coordinate & SN & Coordinate & SN & Coordinate \\
\hline \hline 1 & $(22.18,72.44)$ & 6 & $(25.82,15.50)$ & 11 & $(30.26,50.45)$ \\
\hline 2 & $(20.35,78.14)$ & 7 & $(31.52,18.62)$ & 12 & $(34.89,44.62)$ \\
\hline 3 & $(62.41,65.72)$ & 8 & $(28.13,18.43)$ & 13 & $(55.78,31.56)$ \\
\hline 4 & $(54.86,70.79)$ & 9 & $(89.34,34.30)$ & -- & -- \\
\hline 5 & $(8.06,35.54)$ & 10 & $(77.98,34.63)$ & -- & -- \\
\hline
\end{tabular}

Group 1 To verify the effectiveness of HABC algorithm, HABC algorithm runs for generating a typical solution. As shown in Fig. 7, is a typical solution by adopting HABC algorithm, it can be seen that the typical solution satisfies all constraints, and Fig. 8 displays the distribution of solutions by adopting HABC algorithm.

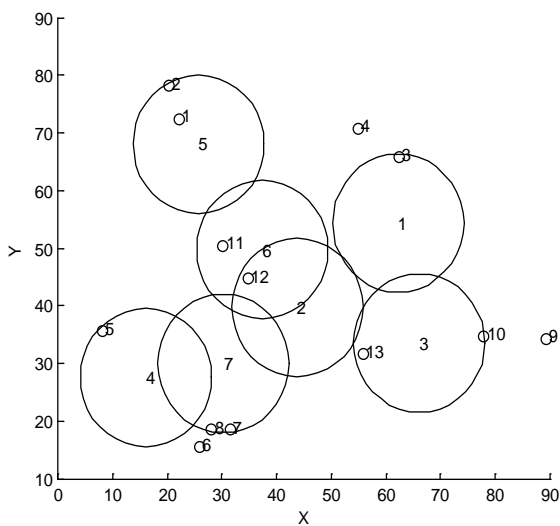

Fig. 7. A typical solution

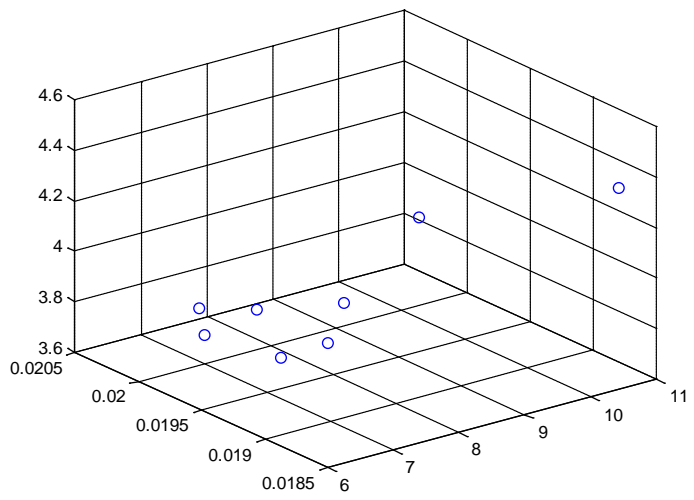

Fig. 8. Distribution of Pareto solutions

Group 2 To verify that the objective function values are affected by key parameters, $R_{1}$ is set to be $9,12,15,18$ and 21 respectively to test the effect of on all objective function values. It can be seen from Fig. 9 that, $R_{1}$ has an important impact on all three objective functions, and with the increase of $R_{1}$, the values of all three objective functions will be better. 

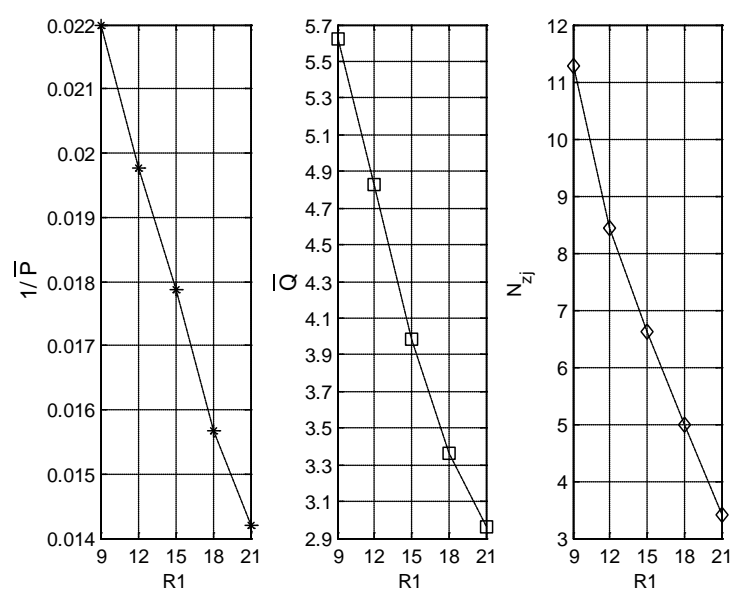

Fig. 9. The influence of $R_{1}$

Group 3 To verify the superiority of HABC algorithm comparing to HPSO algorithm, this paper takes typical performance indexes as comparison items, i.e., coverage index (C-index), evenness index (E-index) and distribution index (D-index), among which C-index and D-index are income type indexes, while E-index is cost type index.

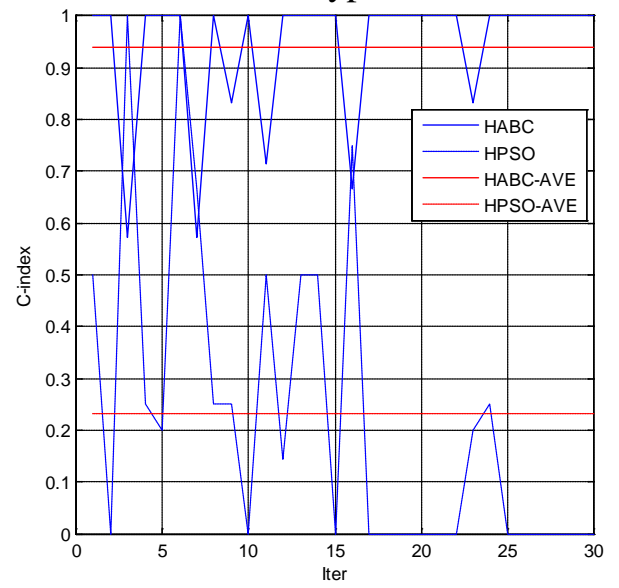

Fig. 10. Comparison result in C-index

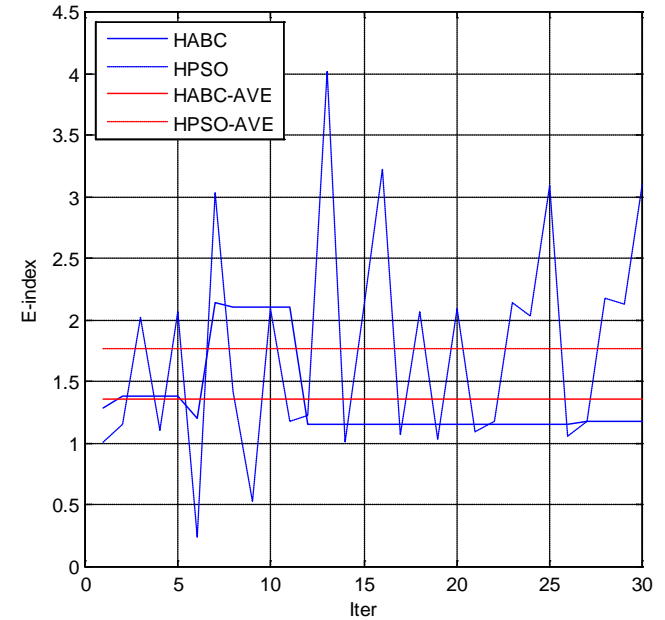

Fig. 11. Comparison result in E-index

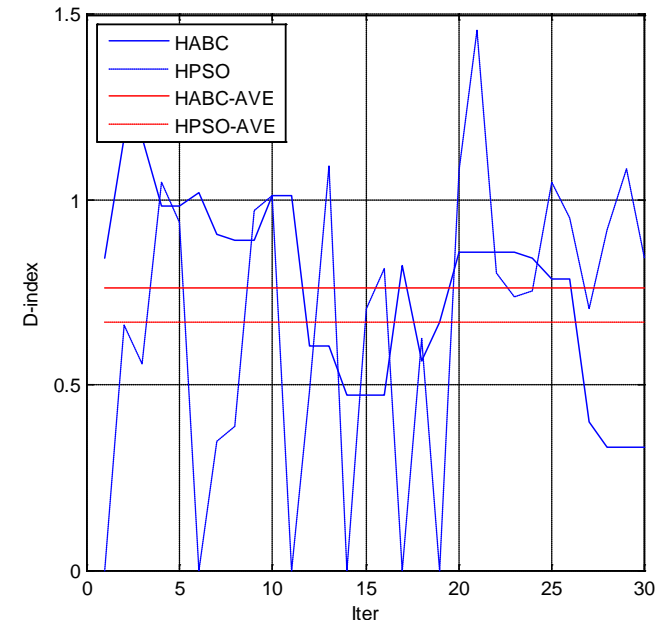

Fig. 12. Comparison result in D-index 
As is shown in Fig. 10, Fig. 11 and Fig. 12, are comparison results of HABC and HPSO algorithm in three indexes, blue polyline represents the change of particular perform index, red line represents the average value of the corresponding perform index. As can be seen, in terms of C-index, HABC is significantly superior to HPSO, and in the aspect of E-index and D-index, HABC is overall better than HPSO.

\section{Conclusion}

MAVs, along with UAVs, to perform combat mission by forming a cooperative engagement system, is the main air engagement pattern in the future, and MAVs and UAVs organized in the form of coalition is an important development direction. One of the key problems is how to provide stable and efficient communication services for all task cluster coalitions, hence, this paper according to the combat characteristic of MAV/UAV cooperative engagement system, and established a bi-level programming model by taking the number of CRNs as upper planning objective, and reciprocal of average communication service quality as well as average number of hops as lower planning objective, and then, it adopted HABC algorithm to solve the model. Next step, it is need to aim at the movement of MAVs and UAVs, and carry out research on dynamic adjustment of CRNs.

\section{Acknowledgment}

This work was supported by National Natural Science Foundation of China (NSFC) under No. 61573017, and Doctoral Foundation of Information and Navigation College, Air Force Engineering Unversity under No. KGD08101604.

\section{References}

[1] OUYANG J, ZHUANG Y, LIN M, "Optimization of beamforming and path planning for UAV-assisted wireless relay networks," Chinese Journal of Aeronautics, vol. 27, no. 2, pp. 313-320, April, 2014. Article (CrossRef Link)

[2] SCHOUWENAARS T, VALENTI M J, FERON E, et al, "Linear Programming and Language Processing for Human-Unmanned Aerial Vehicle Team Missions," Journal of Guidance, Control and Dynamics, vol. 29, no. 2, pp. 303-313, April, 2006. Article (CrossRef Link)

[3] RAISSI D M, CHANDRASHEKAR K, BARAS J S, "UAV placement for enhanced connectivity in wireless ad-hoc networks,” CSHCN Technical Report, 2004. https://ece.umd.edu/ baras/publications/reports/2004/DehkordiCB_TR_2004-34.pdf

[4] WAN L J, YAO P Y, "Aerial Cooperative Combination Formation Method of Manned/ Unmanned Combat Agents," in Proc. of 2013 Chinese Intelligent Automation Conference, pp. 155-164, August 23-25, 2013. Article (CrossRef Link)

[5] ZHONG Y, ZHANG J Y, DENG C L, "Cooperative Engagement Problems About Manned/ Unmanned Aerial Vehicles,” Command Information System and Technology (in Chinese), vol. 8, no. 4, pp. 19-25, August, 2017. Article (CrossRef Link) 
[6] HSIEH M A, COWLEY A, KUMAR R V, et al, "Maintaining network connectivity and performance in robot teams,” Journal of Field Robotics, vol. 25, no. 1-2, pp. 111-131, January, 2008. Article (CrossRef Link)

[7] YAMAMOTO T, NAMATAME A, "Evolutionary Optimized Networks for Consensus and Synchronization,” in proc. of International Conference on Computational Science and Its Applications, pp. 321-335, March 23-26, 2015. Article (CrossRef Link)

[8] LANZA G J M, GOMEZ P J A, VEGA R M A, et al, "A parallel evolutionary approach to solve the relay node placement problem in wireless sensor networks," in Proc. of the 15th Annual Conference on Genetic and Evolutionary Computation, pp. 1157-1164, Jane 6-10, 2013. Article (CrossRef Link)

[9] CHEN G T, CUI S H, "Relay node placement in two-tiered wireless sensor networks with base stations,” Journal of Combinatorial Optimization, vol. 26, no. 3, pp. 499-508, 2013. Article (CrossRef Link)

[10] GUAN Z F, "Current Status and Trend of US Military UA Communication System," Communication Technology (in Chinese), vol. 47, no. 10, pp. 1109-1113, October, 2014. Article (CrossRef Link)

[11] ZAVLANOS M M, SPESIVTSEV L, PAPPAS G J, “A distributed auction algorithm for the assignment problem," in Proc. of the 47th IEEE Conference on Decision and Control, pp. 1212-1217, December 9-11, 2008. Article (CrossRef Link)

[12] ZONG R, GAO X B, PENG J H, "Deployment optimization of the self-organized network on near space platforms based on the game theoretical learning algorithm," Journal of Xidian University (in Chinese), vol. 40, no. 5, pp. 188-193, October, 2013. Article (CrossRef Link)

[13] SENNI P, JOHN S B, CHARLES J G, "Aerial platform placement algorithms to satisfy connectivity, capacity and survivability constraints in wireless ad-hoc networks," in Proc. of IEEE GLOBECOM, pp. 1-7, November 30-December 4, 2008. Article (CrossRef Link)

[14] HAN Z, SWINDLEHURST A. L, LIU K.J. R, "Optimization of MANET connectivity via smart deployment/ movement of unmanned air vehicles," IEEE Transactions on vehicular technology, vol. 58, no. 7, pp. 3533-3546, July, 2009. Article (CrossRef Link)

[15] REN W, Beard R W., "Distributed consensus in multi-vehicle cooperative control: theory and applications”, 1st Edition, Springer, London, 2007. Article (CrossRef Link)

[16] LINDQUIST J M, “An analysis of degraded communications in the army's future force," Naval Postgraduate School, 2009. https://core.ac.uk/download/pdf/36695284.pdf

[17] BURDAKOV O, DOHERTY P, HOLMBERG K, et al, "Optimal placement of UV-based communication relay nodes,” Journal of Global Optimization, vol. 48, no. 4, pp. 511-531, April, 2015. Article (CrossRef Link)

[18] RANGA V, DAVE M, VERMA A K, "Relay node placement to heal partitioned wireless sensor networks,” Computers and Electrical Engineering, vol. 48, no. c, pp. 371-388, November, 2015. Article (CrossRef Link)

[19] CALINESCU G, "Relay placement for two-connectivity," Discrete Optimization, vol. 14, no. 14, pp. 17-33, November, 2014. Article (CrossRef Link) 
[20] HAN B, LI J, SU J S, "Optimal relay node placement for multi-pair cooperative communication in wireless networks," in Proc. of IEEE Wireless Communications \& Networking Conference, pp. 4724-4729, April 7-10, 2013. Article (CrossRef Link)

[21] ZHOU F H, WU Y P, SUN H J, et al, "UAV-Enabled Mobile Edge Computing: Offloading Optimization and Trajectory Design," in Proc. of IEEE ICC 2018, to be published. https://arxiv.org/abs/1802.03906

[22] ISLAM M H, DZIONG Z, SOHRABY K, et al, "Capacity-optimal relay and base station placement in wireless networks," in Proc. of International Conference on Information Networking, pp. 358-363, February 1-3, 2012. Article (CrossRef Link)

[23] ZHONG Y, XIA J B, FU K, “Dynamic cluster-based routing algorithm in DTN,” Application Research of Computers (in Chinese), vol. 32, no. 11, pp. 3393-3398, November, 2015. Article (CrossRef Link)

[24] AAO O, "Stratospheric Propagation and HAPs Channel Modeling," Blekinge Institute of Technology, 2007.

[25] ZHOU F H, WU Y P, LIANG Y C, et al, "State of the Art, Taxonomy, and Open Issues on NOMA in Cognitive Radio Networks," IEEE Wireless Communications, vol. 25, no. 2, pp. 100-108. Article (CrossRef Link)

[26] KARABOGA D, “An idea based on honey bee swarm for numerical optimization,” Erciyes University Technical Report, 2005. https://pdfs.semanticscholar.org/015d/f4d97ed1f541752842c49d12e429a785460b.pdf

[27] BI X J, WANG Y J, “Constraint multi-objective evolutionary algorithm based on artificial bee colony algorithm,” Journal of Jilin University (Engineering and Technology Edition), vol. 43, no. 2, pp. 397-403, April, 2013.

http://www.wanfangdata.com.cn/details/detail.do? type=perio\&id=jlgydxzrkxxb201302022 

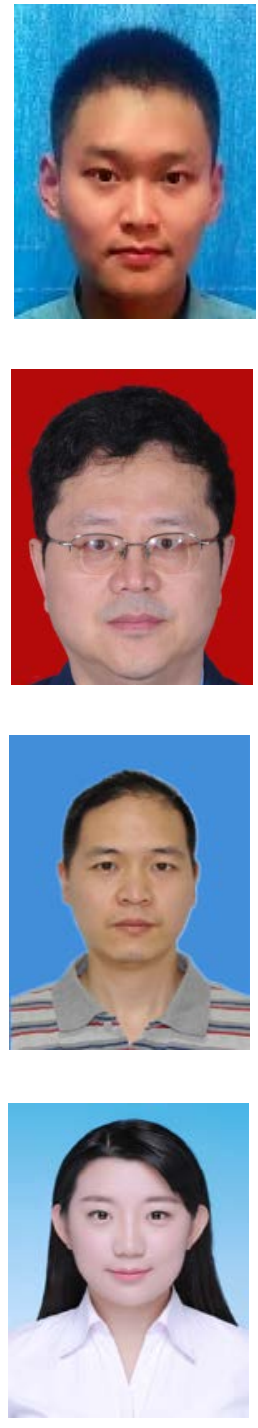

Yun Zhong was born in 1990. He received his B.S. degree in Network Engineering, and M.S. degree in Communication and Information System, from Air Force Engineering University in 2012 and 2014, respectively. He is currently a Ph.D. student of Air Force Engineering University. His research interests include decision support system and MAV/UAV cooperative engagement.

Peiyang Yao was born in 1960. He received his B.S. and M.S. degrees in Communication Engineering from Xi'dian University in 1982 and 1989. After completing his M.S. Program, he worked as a lecturer in Air Force Telecommunications Engineering College. From 1993 to 1999, he worked as an associate professor in Air Force Telecommunications Engineering College. Then, he worked as an associate professor in Air Force Engineering University from 1999 to 2003. Since 2003, he worked as a professor in Air Force Engineering University. His research interest is command information system.

Lujun Wan was born in 1986. He received his B.S. degree in Communication Engineering, and M.S. degree in Communications and Information System, from Air Force Engineering University in 2007 and 2009, respecitively, and he received his Ph.D. degree in Military Informatics from Air Force Engineering University in 2013. After receiving his Ph.D. degree, he worked as a lecturer in Air Force Engineering University. His research interests include operational organization design and air traffic control.

Yeming Xiong was born in 1992. She received her B.S. degree in Information Management and Information System from Xi'dian University in 2014, and M.S. degree in Management Science and Engineering from University of Chinese Academy of Sciences in 2017. She is currently a finance system administrator in Xi'dian University. Her research interest is financial informatization. 